\title{
Study on Application of Diacetylene- containing Copolyurethanes (DA-coPUs) coating as strain sensors using Raman Spectroscopy
}

\author{
Widayani Sutrisno, ${ }^{1, *}$ John L. Stanford, ${ }^{2}$ and Peter A. Lovell ${ }^{2}$ \\ ${ }^{1}$ Department of Physics, Bandung Institute of Technology, Jl. Ganesa 10, Bandung 40132, Indonesia \\ ${ }^{2}$ Manchester Material Science Centre, UMIST, Manchester, United Kingdom
}

\begin{abstract}
A two-phase copolymer DA-coPUs containing of $50 \%$ hard-segment has been prepared using a polyoxypropylene-diol with molecular weight of 2000 (Voranol 2000), 4,4 -diphenylmethane diisocyanate (MDI) and 2,4-hexadiyne-1,6-diol (HDD) via a one-shot, bulk polymerization process. Solution of linear, as-prepared DA-coPUs in N,N-Dimethylacetamide (DMAc) was coated onto pre-heated steel beam. Cross polymerization of the DA-coPUs were carried out using heating under nitrogen at $100^{\circ} \mathrm{C}$ for 5 hours. Deformation micromechanics of the DA-coPUs has been studied using simultaneous 4-point bending testing and Raman spectroscopy. The results showed that the coating has poorer strain- induced Raman band shift factors than that of pure DA-coPUs [1]

(C) 2005 Jurusan Fisika FMIPA ITS
\end{abstract}

KEYWORDS: DA-coPUs Coating, Raman spectroscopy, strain- induced Raman band shift factors

\section{INTRODUCTION}

The objective of the work was to investigate the application of a new DA-coPUs coating as strain sensor using Raman spectroscopy. The basic principle is, as with pure polydiacetylenes, the Raman $-\mathrm{C} \equiv \mathrm{C}-$ stretching bands of polydiacetylene backbones that are located in the hard domains of the DA-coPUs undergo stress-induced band shifts to lower frequency during tensile deformation. Linear shift of Raman peak frequency under tensile deformation was first reported by Mitra et al. [2] who found that the Raman peak frequency of $>\mathrm{C}=\mathrm{C}<$ and $-\mathrm{C} \equiv \mathrm{C}-$ stretching bonds in polydiacetylene single crystal fibers decrease linearly during elongation with the shift factors of about 6 and $19 \mathrm{~cm}^{-1 \%} \%^{-1}$, respectively. This observation illustrated that the macroscopic deformation was directly transformed into the covalent bonds along the polymer backbones. It was thought that the stress-induced Raman band shift correlates with the an-harmonicity of bond constants, where bond force constants depend on the bond length $[2,3]$.

Another DA-coPUs coating for used as strain sensor has been studied. DA-coPUs systems prepared in a bulk using poly(propylene glycol with molecular weight of 400 and 1000) (PPG 400 and PPG 1000), MDI and HDD, were developed by Stanford et al. [4] was demonstrated to show well-defined Raman spectra, and the $-\mathrm{C} \equiv \mathrm{C}-$ stretching

*E-MAIL: widayani@fi.itb.ac.id band of polydiacetylenes in the hard-segment, was shifted to lower frequency when the sample was subjected to tensile deformation. The DA-coPUs waere successfully coated onto aluminium and showed consistency Raman shift factor. In this work, new DA-coPUs system has been synthesized based on a polyoxypropylene-diol with molecular weight of 2000 (Voranol 2000), 4,4'diphenylmethane diisocyanate (MDI) and 2,4-hexadiyne-1,6-diol (HDD) via a one-shot, bulk polymerization process. The reaction of isocyanate with hydroxyl groups gives the formation of urethane linkage as follows:

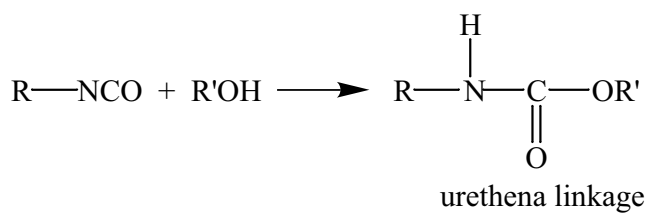

DA-coPUs are known as two-phase copolymers, where both the polyol and diacetylenes react with isocyanates to form soft- and hard-segments, respectively. As shown in figure 1, the chemistry of the DA-coPUs involves two processes. The first process is the formation of linear (asprepared) copolyurethanes that contain diacetylene units in their repeat units. The next process is the crosspolymerization process, where diacetylenes units are transformed to polydiacetylenes chains via a topochemical polymerization process. As with pure polydiacetylenes, DA-coPUs undergo cross- polymerization when subjected to heating or radiation. 


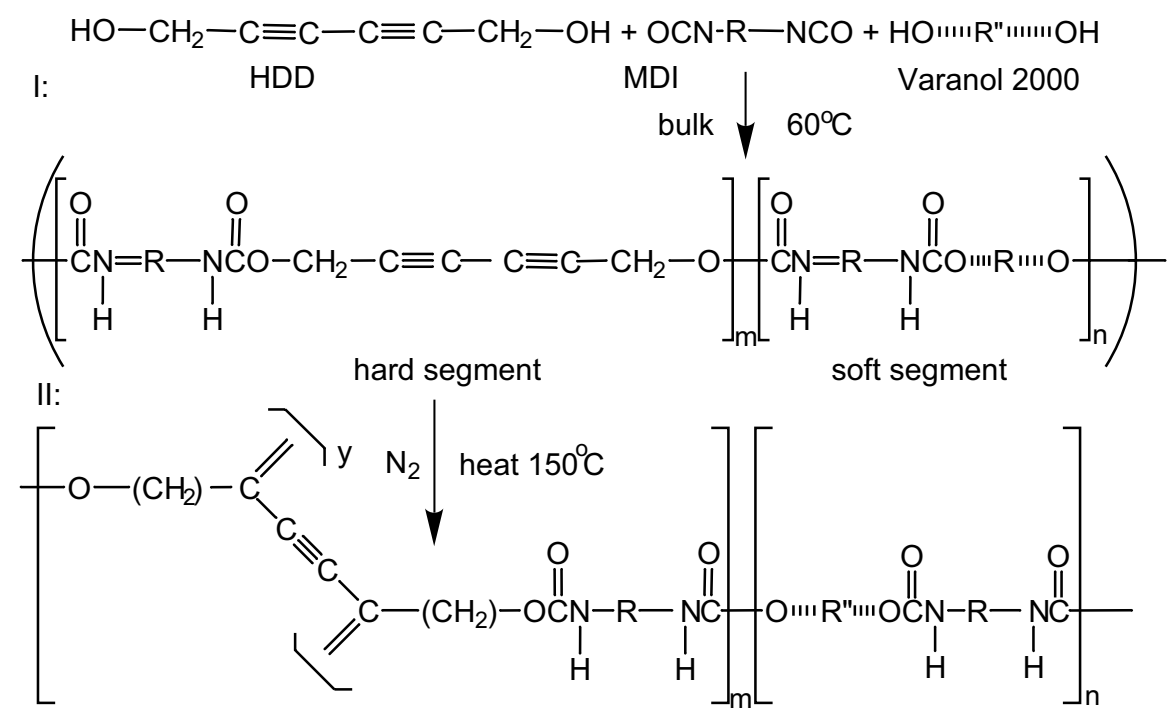

FIG. 1: The chemistry of preparation of cross-polymerized DA-coPUs [4]: I. Formation of linear DA-coPU from 2,4-hexadiyne-1,6diol (HDD), an ethylene oxyde-terminated, polyoxypropylene diol (Voranol EP 2000) and 4,4'-methylene diphenylene diisocyanate (MDI). The DA-coPU contains $50 \%$ by weight of HDD/MDI hard segments. II. Thermal cross-polymerization of diacetylene units in the hard-segment phases of the copolyurethanes.

\section{EXPERIMENT}

The chain extender, HDD, was synthesized according to Hay procedure as reported by Wang, Y-F [5]. The HDD was re-crystallized from ethyl acetate and stored in a sealed bottle wrapped with aluminium foil, which was then placed in a dessiccator and kept in a refrigerator before use. As received MDI (Dow Chemical) was purified by hot-sintering at $60^{\circ} \mathrm{C}$ using a pre-heated grade 4 sinter under a reduced pressure. Clear pure MDI was placed in a sealed plastic bottle and stored at $-20^{\circ} \mathrm{C}$ before use. As-received V-2000 (Dow Chemical) was purified using a rotary-film evaporator at $80^{\circ} \mathrm{C}$ for at least 6 hours. To prevent uptake moisture from atmosphere, the dried V-2000 was stored over $4 \AA \AA$ molecular sieve in a sealed plastic bottle, in a desiccator before use.

Molecular equivalent weights of all the reactants were determined by end-group analysis (EGA). EGA of the hydroxyl functional groups on V-2000 and HDD was carried out using acetic anhydride as the esterification reagent in freshly distilled pyridine. An excess of acetic anhydride was used to force the equilibrium to the product side, with pyridine acting as both solvent and catalyst for the process. After the esterification is completed, the residual acetic anhydride is reacted with water to produce acetic acid in the solution that is then titrated against a strong base, 0.5 $\mathrm{M}$ sodium hydroxide $(\mathrm{NaOH})$ solution, to phenolphthalein end point. As V-2000 may contain residual acid, resulting from its manufacture, so an acidity correction was needed in determining its equivalent weight. Catalyst 2,2 diazabicyclo $[2,2,2]$ was added into acetylating mixture for EGA of HDD and this mixture was isolated from heat and light to prevent topochemical polymerization. The equivalent weight of the MDI was determined by reaction with din-butyl amine $\left(\mathrm{C}_{8} \mathrm{H}_{1} 9 \mathrm{~N}\right)$ in MEK. The amine reacts with the isocyanate group of MDI to give the corresponding trisubstituted urea groups. An excess of amine is used and the unreacted amine was determined by titration with acid, $0.103 \mathrm{M}$ hydrochloride acid $(\mathrm{HCl})$, to bromocresol green end point.

The DA-coPUs with $50 \%$ of HS content were prepared on a scale to give total weights in the range 10 to $50 \mathrm{~g}$ using $1 \%$ molar excess of the isocyanate groups. The DAcoPUs were prepared via a one-shot, bulk polymerization process using the following procedure. First, MDI was accurately weighed into a plastic syringe before being heated

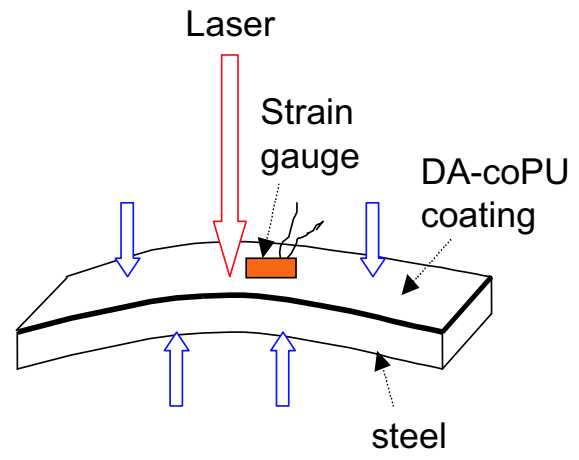

FIG. 2: Schematic diagrams of 4-point bending tests for tension. 
at $60^{\circ} \mathrm{C}$. The required amounts of HDD and V-2000 were mixed at $90^{\circ} \mathrm{C}$ to form a clear mixture (30 minutes). The pre-melted MDI was then injected into the mixture, followed by stirring for 1 minute using a mechanical stirrer. Finally, the complete-stirred mixture was cast into a pre-heated PTFE mould $\left(60^{\circ} \mathrm{C}\right)$ and the solid polymerization continued at $60^{\circ} \mathrm{C}$ for further 150 minutes. The coatings were prepared using a procedure as follows. Solution of $30 \% \mathrm{w} / \mathrm{w}$ as-prepared copolymer in DMAc was coated onto steel beams $(\sim 0.5 \times 10 \times 70) \mathrm{mm}$. After it has been dried at room temperature, the coating was then cross-polymerized by heating under nitrogen at $100^{\circ} \mathrm{C}$ for 5 hours. Deformation micromechanics studies were carried out using simultaneous 4-point bending tests and resonance Raman spectroscopy as shown in Figure 2.

The DA-coPUs Raman spectra were obtained using HeNe laser $(632.8 \mathrm{~nm})$ via a Renishaw Raman $1000 \mathrm{spec}-$ trometer. A modified Nikon optical microscope with a 50 $\times$ objective lens of 0.6 numerical aperture was used to focus the incident laser to a $2 \mu \mathrm{m}$ spot on the specimen.

\section{RESULTS AND DISCUSSION}

DCS curves in Figure 3 show the exotherm peaks of the DA-coPUs for before and after cross-polymerized in the region of 191 to $242^{\circ} \mathrm{C}$. Complex form of the peaks indicates that this exotherm process represent more than only one process, i.e. the peaks may represent both cross- polymerization in the hard-segment regions and degradations. Even the peak area decreases from the as-prepared to the cross-polymerized sample, but it seems that the crosspolymerized sample still contains a significant amount of the non cross-polymerized hard segment. This might influence the effectiveness of strain transfer to the bonds in the polydiacetylenes. The high sensitivity of polydiacetylenes to Raman spectroscopy allows their molecular structures to be studied. Some researchers have reported some differences in Raman shift frequency and intensity that correspond to the differences in molecular structures for some polydiacetylenes. Nitzsche et al. [6] reported the different shapes of both $>\mathrm{C}=\mathrm{C}<$ and $-\mathrm{C} \equiv \mathrm{C}-$ stretching bands of DA-coPUs (PTMO / MDI / HDD) that were prepared by casting using different solvents.

As shown by Figure 4, Raman spectrum of crosspolymerized DA-coPUs coating mainly reflect the polydiacetylene backbone vibrations where the $-\mathrm{C} \equiv \mathrm{C}-$ and $>\mathrm{C}=\mathrm{C}<$ stretching bands give Raman peaks at about 2100 $\mathrm{cm}^{-1}$ and $1480 \mathrm{~cm}^{-1}$, respectively. Studies on vibrational motions of diacetylene polymers using a simple point-mass and harmonic force-constant model showed the two highest frequency vibrational modes at $\sim 2100$ and $1500 \mathrm{~cm}^{-1}$ are essentially backbone bonds vibrations [7]. These 'fingerprints' of the polydiacetylene chains represent the $\nu \sim-\mathrm{C} \equiv \mathrm{C}-$ and $\nu \sim>\mathrm{C}=\mathrm{C}<$ vibrations, respectively. Raman spectra of polydiacetylenes commonly

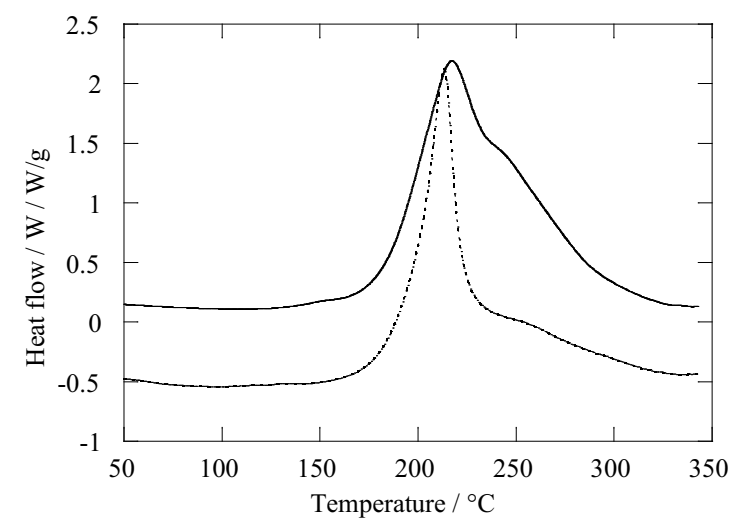

FIG. 3: Exothermal heat flow curves of the DA-coPUs showing how the exothermic peak evolves with heating. The curves are from the DA-coPUs containing $50 \mathrm{wt} \%$ hard-segment content. (-) as-prepared; (-·.) cross-polymerized.

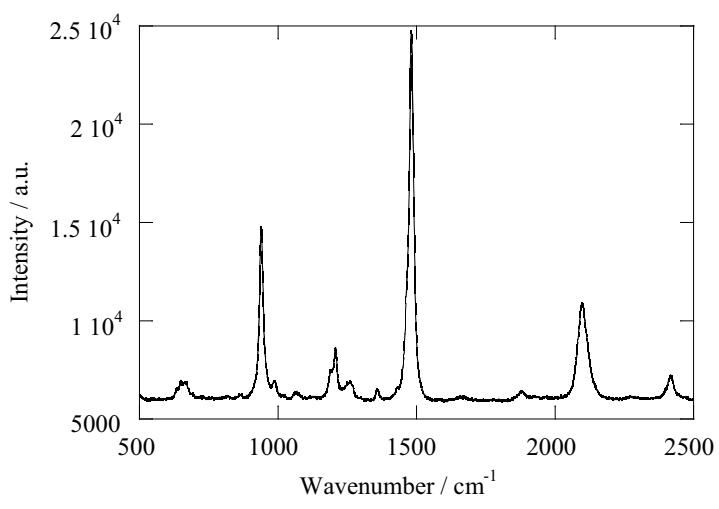

FIG. 4: Spectrum of DA-coPUs coating after crosspolymerization. The peaks at about $2100 \mathrm{~cm}^{-1}$ and $1480 \mathrm{~cm}^{-1}$ are from $>\mathrm{C}=\mathrm{C}<$ and $-\mathrm{C} \equiv \mathrm{C}-$ stretching bands respectively.

reflect two other vibrational motions of polydiacetylenes backbones at frequencies $\sim 1220 \mathrm{~cm}^{-1}$ and $\sim 985 \mathrm{~cm}^{-1}$ that correspond with $\delta \mathrm{C}-\mathrm{C}=\mathrm{C}$ and $\delta \mathrm{C}-\mathrm{C} \equiv \mathrm{C}$, respectively [8].

Figure 5 illustrates a typical distribution of Raman peak position for a non-deformed sample obtained from 100 measurements within area $\sim\left(0.5 \times 1 \mathrm{~cm}^{-2}\right)$. The nonuniformity of the Raman bands signifies that the polydiacetylene chains in the DA-coPUs are randomly oriented. Therefore it is important to focus the laser illumination on the same area during microdeformation-Raman spectroscopy testing.

Figure 6 shows the Raman wavenumber of the $-\mathrm{C} \equiv \mathrm{C}-$ stretching band versus the overall strain under 4-point bending test. Similar with linear shift of Raman peak of $-\mathrm{C} \equiv \mathrm{C}-$ stretching bonds in polydiacetylene single crystal fibers frequency under tensile deformation as reported 


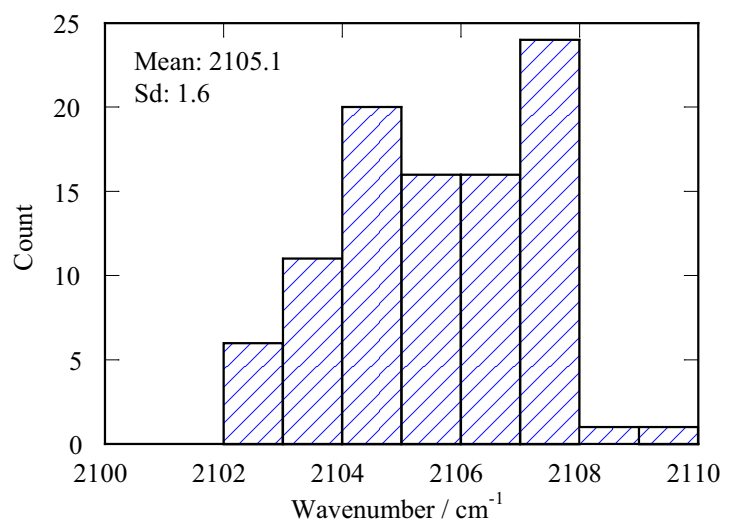

FIG. 5: Typical distribution of Raman peak position based on 100 measurements within $\mathrm{a} \sim 0.5 \times 1 \mathrm{~cm}^{-2}$ area for a nondeformed coating.

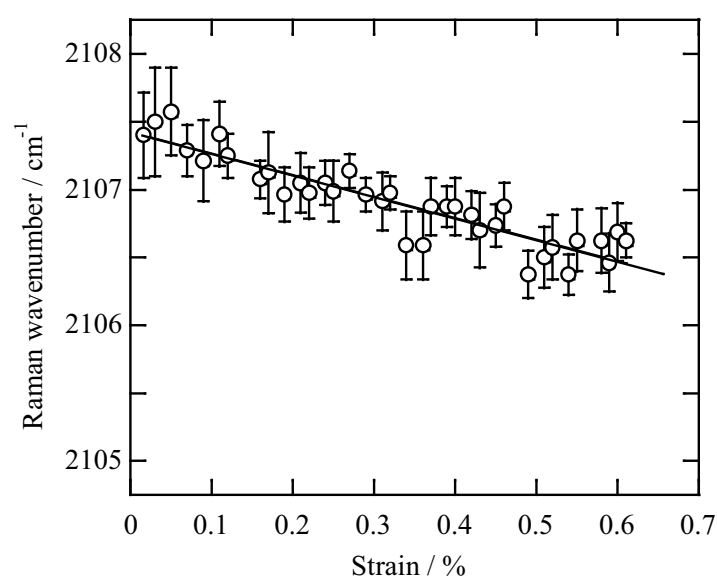

FIG. 6: Plots of the Raman wavenumber of the $-\mathrm{C} \equiv \mathrm{C}-$ stretching band of the DA-coPUs coating versus the overall strain. by Mitra et al. [2], the Raman $-\mathrm{C} \equiv \mathrm{C}-$ stretching band of the DA-coPUs underwent shifts to a lower frequency upon tensile deformation. The shift in band position indicates that the overall mechanical stress is transferred into deformation of polydiacetylene chains in the hard-segment phase of the DA-coPUs coating during testing. The straininduced Raman band shift factors, $S_{\text {epsilon, }}$ can be determined from the slope of the plot, $d(\delta \Delta \nu) / d \epsilon$. Linear regression analysis was performed on the data in order to determine this Raman band shift factor. The shift factor of the DA-coPUs coating was found as $-1.3 \mathrm{~cm}^{-1} \%^{-1}$. This value is lower than that of DA-coPUs bulk that has similar composition, which was reported as $-2.1 \mathrm{~cm}^{-1} \%^{-1}[1]$. The difference may be due to different treatment on the DA-coPUs, both in the synthesis and heating procedure for cross-polymerization process. The other possibility is because of the DA-coPUs might not well-coated on the beam.

\section{CONCLUSION}

The DA-coPUs coating shows a poor shift factor, $d(\delta \Delta \nu) / d \epsilon$, as low as $-1.3 \mathrm{~cm}^{-1} / \%$ strain. This value is lower than that of DA-coPUs bulk that has similar composition, which was reported as $-2.1 \mathrm{~cm}^{-1} \%^{-1}$ [1]. It suggests that the copolymer probably not well coated onto the steel beam. This also may be due to different procedures, both in the synthesis and in cross-polymerization. DSC observation indicates that the sample after heating was not fully cross-polymerized, which may influence the effectiveness of strain transfer to the bonds in the polydiacetylenes.
[1] Sutrisno, W.; Ph. D. Thesis, University of Manchester Institute of Science and Technology, 2003.

[2] Mitra, V. K.; Risen, W. M.; Baughman, R. H.; J. of Chem. Phys., 66, 6, 2731-2736(1977).

[3] Batchelder, D. N.; Bloor, D.; J. of Polym. Sci.: Polym. Phys. Ed., 17, 569-581 (1979).

[4] Stanford, J. L.; Young, R. J.; Day, R. J.; Polymer, 32, 9, 1713-1725 (1991).

[5] Wang, Y-F.; Ph.D. Thesis; The University of Manchester;
1992.

[6] Nitzsche, S. A.; Hsu, S. L.; Hammond, P. T.; Rubner, M. F.; Macromolecules, 25, 2391-2396 (1992).

[7] Lewis, W. F.; Batchelder, D. N.; Chem. Phys. Lett., 60, 2, 232-237 (1979).

[8] Wu, G.; Tashiro, K.; Kobayashi, M.; Macromolecules, 22, 188-196 (1989). 